\title{
Unstable state decay in non-Markovian heat baths and weak signals detection
}

\author{
J. I. Jiménez-Aquino ${ }^{a}$, N. Sánchez-Salas ${ }^{\mathrm{b}}$, L. Ramírez-Piscina ${ }^{\mathrm{c}}$, \\ M. Romero-Bastida ${ }^{\mathrm{d}}$ \\ ${ }^{a}$ Departamento de Física, Universidad Autónoma Metropolitana-Iztapalapa \\ C.P. 09340, México, Distrito Federal \\ ${ }^{b}$ Escuela Superior de Física y Matemáticas, Instituto Politécnico Nacional, \\ Edif. 9 UP Zacatenco, 07738, CDMX, México \\ ${ }^{c}$ Departament de Física, Universitat Politècnica de Catalunya, \\ Avinguda Doctor Marañón 44, 08028 Barcelona, Spain \\ ${ }^{d}$ SEPI ESIME-Culhuacán, Instituto Politécnico Nacional, \\ Delegación Coyoacán, 04430, CDMX, México
}

\begin{abstract}
The statistics of the first passage time and nonlinear relaxation time are used to characterize the decay process of an unstable state for an electrically charged Brownian particle embedded in non-Markovian heat baths under the action of an external electric field. The relaxation process is described, in the overdamped regime, by a Generalized Langevin Equation (GLE) characterized by an arbitrary friction memory kernel and a bistable potential profile. By applying the quasideterministic approach, the statistics of the mean first passage time is calculated through the exact analytical solution of the GLE with arbitrary memory kernel in the linear regime of the bistable potential. To characterize the relaxation process including the nonlinear contributions of the bistable potential, we use the specific Ornstein-Uhlenbeck friction memory kernel to exactly calculate the nonlinear statistics of the mean first passage time as well as the nonlinear relaxation time. Both characteristic times are applied for possible detection of weak signals in the decay process of the unstable state.
\end{abstract}

Email addresses: ines@xanum.uam.mx (J. I. Jiménez-Aquino), norma@esfm.ipn.mx (N. Sánchez-Salas), laure.piscina@upc.edu (L. Ramírez-Piscina), mromerob@ipn.mx (M. Romero-Bastida) 
Keywords: Decay of an unstable state, mean first passage time, nonlinear relaxation time, generalized Langevin equation, weak signals detection PACS: 05.40.-a

\section{Introduction}

The First Passage Time (FPT) is a paradigmatic topic of great interest in science which continues to be applied in a variety of systems as diverse as physics, chemistry, biology, finance, etc. $[1,2,3]$. For instance, it has recently been studied in viscoelastic fluids [4], diffusive processes - ranging from ordinary diffusion (Brownian motion) to continuous-time random walks $[5,6]$ fractional Brownian motion [7, 8], Lévy flights [9, 10], trapped diffusion [11], geometric confinement [3, 12], Ornstein-Uhlenbeck process $[13,14]$, etc. The FPT has also been explored in non-Markovian situations including biological systems $[15,16]$, random walks in confined media $[17,18]$, detection of weak signals [19], among others [20, 21, 22, 23, 24, 25, 26, 27, 28, 29].

In the study of Brownian motion, it is well known that the hypothesis of white noise for the noise correlation function (delta correlation function) is assumed to be valid when both mass and size of the Brownian particle are much larger than the surrounding molecules, and therefore the stochastic process is called Markovian. When the mass and size of the Brownian particle are not larger but roughly the same as the surroundings, the assumption of a delta correlation function for the noise is no longer valid. This means that any physical interaction between the Brownian particle with its surrounding actually takes place within a finite correlation time. In this case, the stochastic process is non-Markovian. To describe the dynamics of a Brownian particle in a more realistic situation Kubo [30], proposed a generalization to the ordinary Langevin equation which is named as Generalized Langevin Equation (GLE).

In 1989 Vemuri and Roy [31] prosed for first time that weak optical signals can be detected via the transient dynamic of a laser system (also known as switch-on process) much in the same way as the superregenerative detection in radar receivers. By transient dynamics, we mean that the laser intensity experiences by spontaneous emission, the decay process from an initial unstable state to its corresponding steady-sate value. The physical idea behind the detection process is that weak signals are greatly amplified when used to trigger the decay process. The criteria proposed in [31] was given in terms of a quantity named as Received-Output (RO) and it could be connected 
with the Nonlinear Relaxation Time (NLRT) in [32]. Beside, another alternative criteria to detect weak optical signals related to the Statistics of the First Passage Time (SFPT) was given in [33]. The experimental execution by measurements the statistics of the initiation time of an argon laser under the influence of an attenuated He-Ne laser was reported in [34].

Over twenty years later, another physical mechanism to detect weak signals was proposed in [35]. It is related to the decay process of an unstable state of a charged Brownian particle under the action of constant crossed electric and magnetic fields.

The mechanism for the detection process in this case is as follows: At initial time $t=0$, the particle is located on the unstable state of the potential profile. Once the decay process is initiated due to the internal fluctuations, a weak external signal is then injected accelerating the decay process. The process also suggests the possible amplification of the weak signal in order to be detected.

The study of relaxation processes of nonequilibrium phenomena through the Mean First Passage Time (MFPT) has been formulated in the context of Langevin, Fokker-Planck, or master equations to Markovian as well as nonMarkovian processes. In particular, the study of the decay process of the unstable states have been focused on different descriptions namely, the evolution of the statistical moments of the relevant variables in terms of Fokker-Planck equations [36, 37, 38, 39], the inverse probability current also in the context of Fokker-Planck equation [40,41], or the study of time evolution of averages in dynamical systems $[42,43,44]$. The works in $[40,41,42,43,44,45]$ provide a good description in the study of such transient stochastic dynamics. However, one of the most appropriate technique in the study of the detection of weak signals in the decay of an unstable state is the so-called Quasideterministic (QD) approach initially proposed in $[36,46]$. It has successfully been connected with the MFPT and NLRT to characterize such a decay process. The QD approach gives a precise physical picture of the mechanism responsible for the decay of the unstable state. The physical mechanism is twofold: Small fluctuations change the initial condition in the surrounding of the unstable state, and afterwards the deterministic motion drives the system out of this state $[22,32,33,35,47]$. Even more, this approach provides a simple way to deal with arbitrary nonlinear unstable potentials without using a Fokker-Planck formulation. On the other hand, practically all the works related to the detection of weak signals in the switch-on process of a laser system rely upon a Langevin-type equation for a complex electric field 
with additive Gaussian white noise. Whereas in the case of a charged Brownian particle in crossed electric and magnetic fields, the standard Langevin equation with constant friction coefficient and additive Gaussian white noise has been used.

In 2014, the problem of the detection of weak signals in the decay process of an unstable state in an Ornstein-Uhlenbeck (OU) heat bath, was studied in the context of a GLE [19]. Two usual theoretical criteria for the detection process were analyzed. One is related to the SFPT and the other, in terms of the RO. To solve the problem the overdamped GLE was significantly reduced to an only one ordinary Langevin equation with Markovian dynamics, with effective parameters depending on a factor incorporating some non-Markovian effects. Our purpose in this work is to study the relaxation process of the decay of the unstable state of a charged Brownian particle embedded in nonMarkovian heat baths, under the action of a constant electric field. Here we propose an alternative strategy allowing to solve the problem in an exact way. In first place, we use the QD approach to calculate the SFPT to characterize such a decay process through the explicit solution of the overdamped GLE with arbitrary friction memory kernel, in the linear approximation of the bistable potential profile. With this general solution we show that the QD approach is an effective theoretical description, even when an arbitrary memory kernel is considered. In this linear regime we obtain the SFPT for a specific OU friction memory kernel.

To characterize the relaxation process of the unstable state taking into account the nonlinear contribution of the bistable potential, we also use the OU memory kernel which allows to achieve our goal by transforming the GLE into two coupled Langevin equations. Working on the nonlinear deterministic equation associated with these two Langevin equations, we are able to characterize such a nonlinear decay process, by means of the nonlinear SFPT and NLRT. As far as we know, the time characterization of the non-Markovian dynamical relaxation of the decay of an unstable state, characterized by a GLE with arbitrary friction memory kernel has not yet been reported in literature.

We also use the two nonlinear time scales to study two theoretical criteria for the detection process of weak signals. Our theoretical results are corroborated by the numerical simulation and compared with the Markovian case. Our work is structured in the following way: In section 2, we calculate the linear SFPT through the explicit solution of the overdamped GLE characterized by an arbitrary friction memory kernel. The consistence of our 
analytical results are verified in the Markovian limit. Explicit results are also obtained in the particular case of an OU memory kernel. Section 3, focuses on explicit and exact analytical calculations of the nonlinear SFPT and NLRT for a specific OU friction memory kernel. These two nonlinear time scales are used in section 4 to study the detection process of weak electric signals; herein all the theoretical results are compared with the numerical simulation. Comments and conclusions are given in section V.

\section{Linear SFPT for the decay of an unstable state in non-Markovian heat baths.}

We consider an electrically charged Brownian particle of mass $m$ and charge $q_{0}$ embedded in a thermal bath of temperature $T$, initially located, at time $t=0$, in an unstable state of a bistable potential $V(x)=-\left(a_{0} / 2\right) x^{2}+$ $\left(b_{0} / 4\right) x^{4}$, being $a_{0}, b_{0}>0$, see Fig. 1 . We are interested in the time characterization of the decay process of such an unstable state for the Brownian particle, taking into account that the physical interaction between the particle with its surroundings is taking place within a finite correlation time. At initial time the relaxation process is triggered by the internal fluctuations and immediately after $(t>0)$ of the decay process, an external electric field is injected accelerating the relaxation process. Because its interaction with its surroundings, the dynamical relaxation of the unstable state is described by the GLE given by

$$
m \dot{v}=-\int_{0}^{t} \gamma\left(t-t^{\prime}\right) v\left(t^{\prime}\right) d t^{\prime}+a_{0} x-b_{0} x^{3}+q_{0} E_{0}+f(t),
$$

where $\gamma(t)$ is the friction memory kernel which is considered as a symmetric function. It is related to the internal noise $f(t)$, which is assumed to be Gaussian with zero mean value and a correlation function that satisfies the fluctuation-dissipation relation of the second kind [30]

$$
\left\langle f(t) f\left(t^{\prime}\right)\right\rangle=k_{B} T \gamma\left(t-t^{\prime}\right),
$$

being $k_{B}$ the Boltzmann constant. To characterize the decay process of the unstable state taking into account an arbitrary friction memory kernel, we just consider the linear approximation of the above nonlinear unstable potential. In this regime of approximation the QD approach provides the relevant information of the mechanism responsible for the decay process. Furthermore, it is around the initial unstable state where the internal fluctuations 


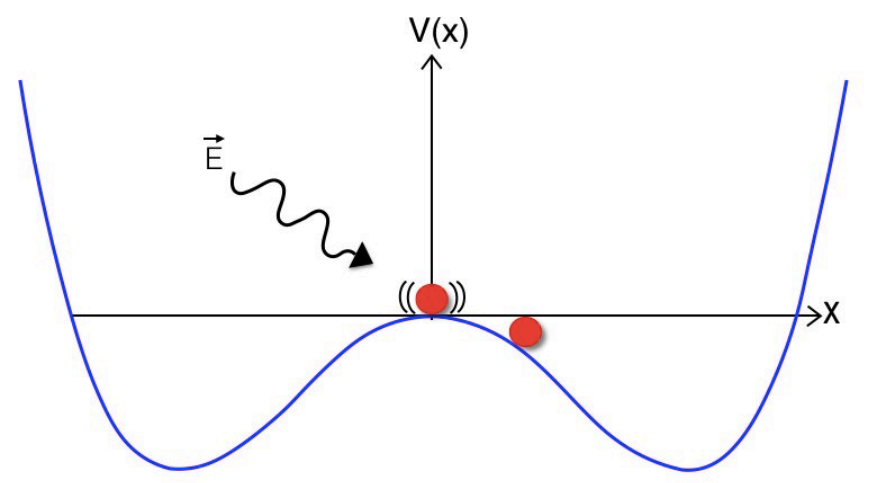

Figure 1: Schematic representation of the decay of the unstable state for a Brownian particle under the action of an external constant electric field.

play a role. Due to these facts, the particle is restricted to move in the interval $-R \leq x \leq R$, where $R$ is the absorbing barrier. In this case, the above GLE must be considered in the overdamped regime such that

$$
\int_{0}^{t} \gamma\left(t-t^{\prime}\right) \dot{x}\left(t^{\prime}\right) d t^{\prime}=a_{0} x+F_{0}+f(t)
$$

being $F_{0}=q_{0} E_{0}$. The solution of this equation by assuming zero initial condition $x(0)=0$, can be obtained using the Laplace transform technique, given as a result

$$
x(t)=\int_{0}^{t} H_{0}\left(t-t^{\prime}\right)\left[F_{0}+f\left(t^{\prime}\right)\right] d t^{\prime},
$$

where $H_{0}(t)$ the Laplace inverse transform of $\hat{H}_{0}(s)$ and

$$
\hat{H}_{0}(s)=\frac{1}{s \hat{\gamma}(s)-a_{0}} .
$$

Also $\hat{\gamma}(s)$ is the Laplace transform of $\gamma(t)$. To apply the QD approach we write the solution (4) in an appropriate form given by

$$
x(t)=h(t) e^{\mathcal{A} t},
$$

where $\mathcal{A}$ must be a positive constant which will depend on the type of model, and thus

$$
h(t)=\int_{0}^{t} e^{-\mathcal{A} t} H_{0}\left(t-t^{\prime}\right)\left[F_{0}+f\left(t^{\prime}\right)\right] d t^{\prime} .
$$


According to the QD approach, the stochastic process $h(t)$ must be a Gaussian random variable in the large-time limit. This is indeed the case if, in this limiting case, the arbitrary function $e^{-\mathcal{A} t} H_{0}\left(t-t^{\prime}\right)$ is convergent and dominates over both the small amplitudes of the external force and small noise intensity. In this case, we expect that $h(\infty)=h=$ cst, is a constant, such that

$$
h=\lim _{t \rightarrow \infty} \int_{0}^{t} e^{-\mathcal{A} t} H_{0}\left(t-t^{\prime}\right)\left[F_{0}+f\left(t^{\prime}\right)\right] d t^{\prime} .
$$

The variance of $h$ is obtained from $\sigma^{2}=\lim _{t \rightarrow \infty} \sigma^{2}(t)$, where $\sigma^{2}(t)=\left\langle h^{2}(t)\right\rangle-$ $\langle h(t)\rangle^{2}$, and thus

$$
\sigma^{2}=\lim _{t \rightarrow \infty} \int_{0}^{t} \int_{0}^{t} e^{-2 \mathcal{A} t} H_{0}\left(t-t^{\prime}\right) H_{0}\left(t-t^{\prime \prime}\right)\left\langle f\left(t^{\prime}\right) f\left(t^{\prime \prime}\right)\right\rangle d t d t^{\prime} .
$$

Using Eqs. (2) and (5), it can be shown that

$$
\sigma^{2}=k_{B} T \lim _{t \rightarrow \infty} e^{-2 \mathcal{A} t}\left[2 \int_{0}^{t} H_{0}\left(t^{\prime} d t^{\prime}+a_{0}\left(\int_{0}^{t} e^{-\mathcal{A} t} H_{0}\left(t^{\prime}\right) d t^{\prime}\right)^{2}\right] .\right.
$$

Therefore, in the large-time limit the process $x(t)$ becomes quasi-deterministic such that

$$
x^{2}(t)=h^{2} e^{2 \mathcal{A} t} .
$$

The random passage time required for the charged particle to reach the potential barrier $R^{2}$ becomes

$$
t=\frac{1}{2 \mathcal{A}} \ln \left(\frac{R^{2}}{h^{2}}\right)
$$

The statistics of this passage time distribution can be calculated from both the Gaussian probability density (GPD) $P(h)$ and Moment Generating Function (MGF) defined by $G(2 a \nu)=\left\langle e^{-2 \mathcal{A} \nu t}\right\rangle=\left\langle\left(R^{2} / h^{2}\right)^{-\nu}\right\rangle$. The GPD reads as

$$
P(h)=\frac{1}{\sqrt{2 \pi \sigma^{2}}} e^{(h-\langle h\rangle)^{2} / 2 \sigma^{2}},
$$

where $\sigma^{2}$ is the same as Eq. (10) and the average $\langle h\rangle$ is given by

$$
\langle h\rangle=F_{0} \lim _{t \rightarrow \infty} \int_{0}^{t} e^{-\mathcal{A} t} H_{0}\left(t-t^{\prime}\right) d t^{\prime} .
$$


Using Eq. (13), it is possible to show that the MGF reads

$$
G(2 \mathcal{A} \nu)=G_{0}(2 \mathcal{A} \nu) e^{-\beta^{2}} M\left(\nu+1 / 2,1 / 2 ; \beta^{2}\right),
$$

with $G_{0}(2 \mathcal{A} \nu)=\left(R^{2} / 2 \sigma^{2}\right)^{-\nu} \Gamma(\nu+1 / 2) / \sqrt{\pi}$ being the MGF in the absence of the external electric force, $M(a, b ; z)$ the Kummer confluent hypergeometric function [55], and $\beta^{2}=\langle h\rangle^{2} / 2 \sigma^{2}$. Given the MGF, the statistics of the passage time is now easy to obtain. In this case, $\langle 2 \mathcal{A} t\rangle=-d G(2 \mathcal{A} \nu) /\left.d \nu\right|_{\nu=0}$ and $\left\langle(2 \mathcal{A} t)^{2}\right\rangle=d^{2} G(2 \mathcal{A} \nu) /\left.d \nu^{2}\right|_{\nu=0}$. After some algebra we can show that the MFPT for arbitrary friction memory kernel, becomes

$$
\langle t\rangle_{L}=\langle t\rangle_{0 L}-\frac{1}{2 \mathcal{A}} e^{-\beta^{2}} \sum_{n=1}^{\infty}[\psi(n+1 / 2)-\psi(1 / 2)] \frac{\left(\beta^{2}\right)^{n}}{n !},
$$

where

$$
\langle t\rangle_{0 L}=\frac{1}{2 \mathcal{A}} \ln \left(\frac{R^{2}}{2 \sigma^{2}}\right)-\frac{1}{2 \mathcal{A}} \psi(1 / 2),
$$

is the MFPT in the absence of the external force. Besides, the function $\psi(x)$ also satisfies the identity $\psi(n+1 / 2)=\psi(1 / 2)+2 \sum_{k=1}^{n} 1 /(2 k-1)$, being $\psi(1 / 2)=-1.96351$. The variance defined by $\left\langle(2 \mathcal{A} \Delta t)^{2}\right\rangle \equiv\left\langle(2 \mathcal{A} t)^{2}\right\rangle-\langle 2 \mathcal{A} t\rangle^{2}$ is shown to be

$$
\begin{aligned}
& \left\langle(2 \mathcal{A} \Delta t)^{2}\right\rangle=\psi^{\prime}(1 / 2)+e^{-\beta^{2}} \sum_{n=1}^{\infty}\left\{[\psi(n+1 / 2)-\psi(1 / 2)]^{2}+\right. \\
& \left.\left[\psi^{\prime}(n+1 / 2)-\psi^{\prime}(1 / 2)\right]\right\} \frac{\beta^{2^{n}}}{n !}-\left\{e^{-\beta^{2}} \sum_{n=1}^{\infty}[\psi(n+1 / 2)-\psi(1 / 2)] \frac{\beta^{2 n}}{n !}\right\}^{2},
\end{aligned}
$$

where also $\psi^{\prime}(n+1 / 2)=\psi^{\prime}(1 / 2)-4 \sum_{k=1}^{n} 1 /(2 k-1)^{2}$, with $\psi^{\prime}(1 / 2)=\pi^{2} / 2=$ 4.934 [55].

\subsection{Markovian case}

Let us now show that the above non-Markovian theoretical description is consistent with the Markovian case, for which the time-dependent friction is memoryless and given by $\gamma(t)=\gamma_{0} \delta(t)$, being $\gamma_{0}$ the friction coefficient. It is easy to show that the Laplace inverse function $H_{0}(t)=\left(1 / \gamma_{0}\right) e^{a t}$ where $a=$ $a_{0} / \gamma_{0}$ and thus $e^{-\mathcal{A t}} H_{0}\left(t-t^{\prime}\right)=\left(1 / \gamma_{0}\right) e^{(a-A) t-a t^{\prime}}$. To ensure the convergence of the function $e^{-\mathcal{A t}} H_{0}\left(t-t^{\prime}\right)$, it must be required that $\mathcal{A}=a$ and thus 
$e^{-\mathcal{A} t} H_{0}\left(t-t^{\prime}\right)=\left(1 / \gamma_{0}\right) e^{-a t^{\prime}}$. So that, Eqs. (10) and (14) in this Markovian case become respectively $\sigma_{M}^{2}=D / a$ and $\langle h\rangle_{M}=F_{0} / a_{0}$, with $D=k_{B} T / \gamma_{0}$ the Einstein diffusion coefficient. The external parameter becomes $\beta_{M}^{2}=$ $\langle h\rangle^{2} / 2 \sigma^{2}=F^{2} / 2 a D$, with $F=F_{0} / \gamma_{0}$. Hence in the Markovian limit and according to Eq. (16), the MFPT reads

$$
\langle t\rangle_{L}^{M}=\langle t\rangle_{0 L}^{M}-\frac{1}{2 a} e^{-\beta_{M}^{2}} \sum_{n=1}^{\infty}[\psi(n+1 / 2)-\psi(1 / 2)] \frac{\left(\beta_{M}^{2}\right)^{n}}{n !},
$$

with

$$
\langle t\rangle_{0 L}^{M}=\frac{1}{2 a} \ln \left(\frac{a R^{2}}{2 D}\right)-\frac{1}{2 a} \psi(1 / 2) .
$$

The variance is the same as Eq. (18), except that the parameters $\mathcal{A}$ and $\beta^{2}$ must be replaced by $a$ and $\beta_{M}^{2}$, respectively.

\subsection{Ornstein-Uhlenbeck friction memory kernel}

In this section, we apply the above theoretical results to the case of an OU friction memory kernel defined by $\gamma\left(t-t^{\prime}\right)=\left(\gamma_{0} / \tau\right) e^{-\left|t-t^{\prime}\right| / \tau}$, where $\gamma_{0}$ is the friction coefficient and $\tau$ the noise correlation time. In this case it is easy to show that the Laplace inverse transform of $\hat{H}_{0}(s)$ given by Eq. (5) becomes

$$
H_{0}(t)=\frac{\tau}{\gamma_{0}(1-a \tau)} \delta(t)+\frac{1}{\gamma_{0}(1-a \tau)^{2}} e^{\mathcal{B} t},
$$

where $\mathcal{B}=a /(1-a \tau)$, which is positive if $1-a \tau>0$ and also $a=a_{0} / \gamma_{0}$. In this case the solution given by Eq. (6) now reads $x(t)=h(t) e^{\mathcal{B} t}$, where $h(t)$ becomes

$$
\begin{aligned}
h_{\text {OU }}(t) & =\frac{\tau}{\gamma_{0}(1-a \tau)}\left[F_{0}+f(t)\right] e^{-\mathcal{B} t} \\
& +\frac{1}{\gamma_{0}(1-a \tau)^{2}} \int_{0}^{t} e^{-\mathcal{B} t^{\prime}}\left[F_{0}+f\left(t^{\prime}\right)\right] d t^{\prime} .
\end{aligned}
$$

Evidently in the large-time limit and also for small values of both the noise intensity and amplitude of the external force, we can see that $h_{O U}(\infty)=h_{O U}$ becomes a Gaussian random variable such that

$$
h_{\text {OU }}=\frac{1}{\gamma_{0}(1-a \tau)^{2}} \int_{0}^{\infty} e^{-\mathcal{B} t}\left[F_{0}+f(t)\right] d t .
$$


Its average value reads

$$
\langle h\rangle_{O U}=\frac{F}{a(1-a \tau)}=\frac{F_{e}}{a},
$$

where $F_{e}=F /(1-a \tau)$. The variance can be calculated from Eq. (10), or from Eqs. (23) and (24). In any case it can be shown that

$$
\sigma_{O U}^{2}=\frac{D}{a(1-a \tau)^{2}}=\frac{D_{e}}{a}
$$

where $D_{e}=D /(1-a \tau)^{2}$ is an effective Einstein diffusion coefficient. The $\beta^{2}$ parameter in this case reads

$$
\beta_{O U}^{2}=\frac{\langle h\rangle_{O U}^{2}}{2 \sigma_{O U}^{2}}=\frac{F^{2}}{2 a D}
$$

which is exactly the same as obtained in the Markovian case, that is, $\beta_{O U}^{2}=$

$\beta_{M}^{2}$. This means that, for the OU process the external parameter $\beta_{O U}^{2}$ does not depend on the heat bath memory effects. The MFPT then reads

$$
\langle t\rangle_{L}^{O U}=\langle t\rangle_{0 L}^{O U}-\frac{1}{2 \mathcal{B}} e^{-\beta_{M}^{2}} \sum_{n=1}^{\infty}[\psi(n+1 / 2)-\psi(1 / 2)] \frac{\left(\beta_{M}^{2}\right)^{n}}{n !},
$$

where

$$
\langle t\rangle_{O L}^{O U}=\frac{1}{2 \mathcal{B}} \ln \left(\frac{a R^{2}}{2 D_{e}}\right)-\frac{1}{2 \mathcal{B}} \psi(1 / 2) .
$$

The variance is also the same as Eq. (18), but the parameter $\mathcal{A}$ must be replaced by the parameter $\mathcal{B}$. It should be noticed that the dimensionless time scale $\langle 2 \mathcal{B} t\rangle_{L}^{O U}$ is the same as the Markovian dimensionless time scale $\langle 2 a t\rangle_{L}^{M}$ given by Eq. (19), if the Einstein diffusion coefficient $D$ in Eq. (20), is replaced by $D_{e}$.

\section{Nonlinear time scales for the decay of an unstable state in Ornstein-Uhlenbeck heat baths.}

For the OU model it is possible to use the QD approach to characterize the decay process of the unstable state taking into account the nonlinear contribution of the bistable potential profile. In this section we will obtain 
the SFPT and the NLRT for the specific OU friction memory kernel. To achieve the goal we introduce the definition of the deterministic dynamic associated with the GLE given by (1). For such a purpose, we define the change of variables defined by

$$
\eta(t)=-\frac{\gamma_{0}}{\tau} \int_{0}^{t} e^{-\frac{\left(t-t^{\prime}\right)}{\tau}} v\left(t^{\prime}\right) d t^{\prime}+f(t)
$$

and

$$
f(t)=\frac{\sqrt{\lambda}}{\tau} \int_{0}^{t} e^{-\frac{\left(t-t^{\prime}\right)}{\tau}} \xi\left(t^{\prime}\right) d t^{\prime},
$$

which allows writing the GLE (1) into the following two coupled quasiMarkovian Langevin equations

$$
\begin{aligned}
m \dot{v} & =a_{0} x-b_{0} x^{3}+F_{0}+\eta(t), \\
\dot{\eta} & =-\frac{1}{\tau} \eta-\frac{\gamma_{0}}{\tau} v+\frac{\sqrt{\lambda}}{\tau} \xi(t),
\end{aligned}
$$

being $\lambda=\gamma_{0} k_{B} T$ and $\xi(t)$ a Gaussian white noise with zero mean value and correlation function $\left\langle\xi(t) \xi\left(t^{\prime}\right)\right\rangle=2 \delta\left(t-t^{\prime}\right)$. In the overdamped regime and in the absence of the external force $F_{0}$, the deterministic dynamic associated with Eqs. (31) and (32) for the variable $\mathfrak{r}=x^{2}$, can be written as

$$
\frac{d \mathfrak{r}}{d t}=\frac{\mathfrak{r}\left(\mathfrak{r}_{s t}-\mathfrak{r}\right)}{C_{0}+(3 / 2) \tau \mathfrak{r}}
$$

where $C_{0}=\mathfrak{r}_{s t} / 2 \mathcal{B}$, and $\mathfrak{r}_{s t}=x_{s t}^{2}=a_{0} / b_{0}$.

\subsection{Nonlinear statistics of the first passage time}

The definition of this time scale, according to the QD approach, is given by [19]

$$
\begin{aligned}
\langle t\rangle_{N L}^{O U} & =\left\langle\int_{h^{2}}^{R^{2}} \frac{C_{0}+\mathfrak{r} g(\mathfrak{r})}{\mathfrak{r}\left(\mathfrak{r}_{s t}-\mathfrak{r}\right)} d \mathfrak{r}\right\rangle=\frac{1}{2 \mathcal{B}}\left\langle\ln \left(\frac{R^{2}}{h^{2}}\right)\right\rangle+\frac{1}{2 \mathcal{B}} \ln \left(\frac{1}{1-M^{2}}\right) \\
& +\frac{3}{2} \tau \ln \left(\frac{1}{1-M^{2}}\right),
\end{aligned}
$$

where $h$ plays the role of an effective initial condition, $R$ is the potential barrier and $M^{2}=R^{2} / x_{s t}^{2}$, such that $0<M^{2}<1$. This time scale now 
becomes

$$
\langle t\rangle_{N L}^{O U}=\langle t\rangle_{0 N L}^{O U}-\frac{1}{2 \mathcal{B}} e^{-\beta_{M}^{2}} \sum_{n=1}^{\infty}[\psi(n+1 / 2)-\psi(1 / 2)] \frac{\left(\beta_{M}^{2}\right)^{n}}{n !},
$$

where

$$
\langle t\rangle_{0 N L}^{O U}=\frac{1}{2 \mathcal{B}} \ln \left(\frac{a R^{2}}{2\left(1-M^{2}\right) D_{e}}\right)-\frac{1}{2 \mathcal{B}} \psi(1 / 2)+\frac{3}{2} \tau \ln \left(\frac{1}{1-M^{2}}\right),
$$

is the nonlinear passage time in the absence of the external electric field. The variance is again the same as Eq. (18).

Although mathematically the form of Eqs. (35) and (36) are very similar to those obtained in [19] [see Eqs. (37) and (38)], the differences between them are basically the new contribution given by the third term of Eq. (36), the effective Einstein diffusion coefficient $D_{e}$, and the external parameter $\beta^{2}$. For instance, this third term is strictly non-Markovian and comes from the nonlinear effects of the particle dynamic, contrary to what happens in Eq. (38) of Ref. [19], in which such a nonlinear contribution is equal to zero. In the present work the Einstein diffusion coefficient is rescaled by the factor $1 /(1-a \tau)^{2}$, whereas in [19], such a factor is $1 /(1-a \tau)$. In this paper the external parameter $\beta^{2}=\langle h\rangle^{2} / 2 \sigma^{2}$, when applied to OU memory kernel, curiously becomes the same as in the Markovian case, that is $\beta_{M}^{2}=F^{2} / 2 a D$, even when $\langle h\rangle_{O U}$ and $\sigma_{O U}^{2}$ are heat bath dependent. However, in [19] such an external parameter is given by $\beta_{e}^{2}=\beta_{M}^{2}(1-a \tau)$, which means a rescaling of the Markovian parameter by the factor $(1-a \tau)$.

All of those differences arise because in our present work, the problem, when applied to the OU friction memory kernel, the overdamped Langevin equation is exactly solved.

It is also interesting to comment that, when the absorbing barrier is removed, the point $x$ can cross the point $x= \pm R$ any number of times and in any direction; in this case a study in terms of the inverse probability current must be performed as done in [40], for Markovian processes. This study leads to an unexpected effect called noise delayed decay (NDD), wherewith the stochastic fluctuations can considerably increase the decay time of unstable and metastable states. The method has been developed to calculate the NLRT for any fluctuation intensity and arbitrary potential profile also in the Markovian case. In the particular case of small fluctuations, the NLRT coincides with the MFPT. Indeed, the NLRT defined in Ref. [40] has been 
calculated for the symmetric bistable potential and other potential profiles. For symmetric bistable potential it has been shown that, for small noise intensity such that $q \ll \Phi\left(x_{m}\right)$, the NLRT given by Eq. (15) of Ref. [40] coincides with the MFPT calculated by Haake et al. [37] and given in Eq. (16) of the same Ref. [40], as expected. The comparison is shown in Fig. 9 of Ref. [40]. In the Markovian case, our Eq. (36) is exactly the same as the MFPT obtained in [37] if the noise parameter $q=k_{B} T$ and $x_{m}= \pm \sqrt{a / b}$, with $\Phi\left(x_{m}\right)=-a^{2} / 4 b$ as the depth of the potential profile. In conclusion, when the QD approach is used to characterize, by means of the MFPT, the Markovian or non-Markovian decay process of an unstable state, this relaxation process is bounded by fixed and absorbing barriers, so that the inverse probability current becomes negligible.

\subsection{Nonlinear relaxation time}

The other time scale used to characterize the dynamical relaxation of the decay of an unstable state is named as, Non Linear Relaxation Time (NLRT) [19]. In the case studied in this work it is associated with the dynamical relaxation of the average $\left\langle x^{2}(t)\right\rangle$, where $x$ is the position of the Brownian particle. The quantity $\langle\cdots\rangle$ stands for the average taken on both, the noise realizations and initial conditions. The quantity $\left\langle x^{2}(t)\right\rangle=\langle\mathfrak{r}(t)\rangle$ evolves from an initial value $\left\langle x^{2}(0)\right\rangle=\langle\mathfrak{r}(0)\rangle$ to its corresponding steady-state value $\left\langle x^{2}\right\rangle_{s t}=\left\langle\mathfrak{r}_{s t}\right\rangle$. The NLRT is defined as

$$
T=\int_{0}^{\infty} \frac{\left\langle x^{2}(t)\right\rangle-\left\langle x^{2}\right\rangle_{s t}}{\left\langle x^{2}(0)\right\rangle-\left\langle x^{2}\right\rangle_{s t}} d t=\int_{0}^{\infty} \frac{\langle\mathfrak{r}(t)\rangle-\langle\mathfrak{r}\rangle_{s t}}{\langle\mathfrak{r}(0)\rangle-\langle\mathfrak{r}\rangle_{s t}} d t
$$

The difference of this time scale with the one given by Eq. (34) is that the NLRT can characterize the complete dynamical relaxation of the unstable state, taking into account the steady-state value $x_{s t}^{2}$ of the potential, whereas in the MFPT Eq. (34) this steady-state value for the $R^{2}=M^{2} x_{s t}^{2}$ is prohibited, since $0<M^{2}<1$. In this case it must be clear that the nonlinear MFPT must be lees that the NLRT.

The connection between the NLRT and the QD approach can be achieved by assuming that $\mathfrak{r}(0) \equiv h^{2}$ is a random variable, which plays the role of an effective initial condition responsible for the decay of the unstable state towards its steady-state value $\mathfrak{r}_{\text {st }}$. By assuming that $\langle\mathfrak{r}(0)\rangle=0$ and substituting Eq. (33) into (37), we get

$$
T_{N L}^{O U}=\frac{1}{2 \mathcal{B}}\left\langle\ln \left(\frac{\mathfrak{r}_{s t}}{h^{2}}\right)\right\rangle+\frac{3}{2} \tau,
$$


where the additional factor $3 \tau / 2$ comes from the nonlinear character of the bistable potential. Alternatively we can write

$$
T_{N L}^{O U}=\frac{1}{2 \mathcal{B}}\left\{\ln \left(\frac{x_{s t}^{2}}{2 \sigma^{2}}\right)-\left\langle\ln \left(\frac{h^{2}}{2 \sigma^{2}}\right)\right\rangle\right\}+\frac{3}{2} \tau
$$

where the variance $\sigma^{2}$ is the same as Eq. (25). For the second term of the right hand side of Eq. (39) we define $I=\left\langle\ln \left(h^{2} / 2 \sigma^{2}\right)\right\rangle$, which can be calculated with the help of the probability density $P(h)$ given by Eq. (13), yielding to

$$
\begin{aligned}
I & =e^{-\beta^{2}} \psi(1 / 2) \\
& +\frac{e^{-\beta^{2}}}{\sqrt{\pi}} \sum_{n=1}^{\infty} \psi(n+1 / 2) \Gamma(n+1 / 2) \frac{(2 \beta)^{2 n}}{(2 n) !}
\end{aligned}
$$

being $\beta^{2}$ the same external parameter defined before. Using the identity $\Gamma(n+1 / 2)=\sqrt{\pi}(2 n-1) ! ! / 2^{n}$, it can also be shown that

$$
I=\psi(1 / 2)+e^{-\beta^{2}} \sum_{n=1}^{\infty}[\psi(n+1 / 2)-\psi(1 / 2)] \frac{\left(\beta^{2}\right)^{n}}{n !} .
$$

Upon substitution into Eq. (39), we finally get the NLRT

$$
T_{N L}^{O U}=T_{0 N L}^{O U}-\frac{1}{2 \mathcal{B}} e^{-\beta_{M}^{2}} \sum_{n=1}^{\infty}[\psi(n+1 / 2)-\psi(1 / 2)] \frac{\left(\beta_{M}^{2}\right)^{n}}{n !}
$$

where

$$
T_{\text {ONL }}^{O U}=\frac{1}{2 \mathcal{B}} \ln \left(\frac{a x_{s t}^{2}}{2 D_{e}}\right)-\frac{1}{2 \mathcal{B}} \psi(1 / 2)+\frac{3}{2} \tau,
$$

is the NLRT in the absence of the external force. In a similar way as in the MFPT, the time scales (42) and (43), are very similar to those obtained in [19] [see Eqs. (26) and (27) ], except for the third term given in Eq. (43) coming from the nonlinear effects of the particle dynamic. In Eq. (27) of Ref. [19], the nonlinear contribution is simply equal to zero.

\section{Weak signals detection}

We now use the two time scales obtained in the case of OU memory kernel to study possible detection of weak signals. For weak signals we mean the 
case when the amplitude of the injected electric field is less or of the same order than the noise intensity which in turn is also small. We first study the criterion related to the SFPT and then the criterion related to the Received Output (RO).

\subsection{Criterion related to SFPT}

This criterion allows determining a critical value of the $\beta$ parameter for which the statistics of the first passage time satisfies

$$
\left[\langle t\rangle_{\beta_{c}}-\langle t\rangle_{\beta=0}\right]^{2} \geq\left\langle(\Delta t)^{2}\right\rangle_{\beta=0} .
$$

If we substitute Eqs. (35) and (18) into this criterion we get

$$
\begin{aligned}
& {\left[\frac{1}{2 \mathcal{B}} e^{-\beta_{M}^{2}} \sum_{n=1}^{\infty}[\psi(n+1 / 2)-\psi(1 / 2)] \frac{\left(\beta_{M}^{2}\right)^{n}}{n !}\right]^{2} } \\
\geq & {\left[\psi^{\prime}(1 / 2)\right]^{1 / 4} . }
\end{aligned}
$$

This result determines a critical value $\beta_{c}=1.36543$ at which the weak signal can be detected. Below this critical value there is no detection and above this value the detection process must be efficient. Notice however that $\beta_{O U}^{2}=\beta_{M}^{2}=F^{2} / 2 a D$, and therefore the critical value is heat bath memory independent and leads to the same result as in the Markovian case, contrary to the prediction from Ref. [19]. It must be noticed also that the same result (45) is obtained if we use the linear time scale (27) and the variance (18).

\subsection{Criterion related to $R O$}

The other criterion used to detect weak signals is the RO which is defined as the ratio $\mathfrak{R}=A_{e} / A_{0}$, where, $A_{e}$ is the area under the curve $\left\langle x^{2}(t)\right\rangle$, when the particle dynamic is under the action of an external electric field, and $A_{0}$ the corresponding area in the absence of the electric field. Accordingly, the RO can be expressed in terms of the time scales given by Eqs. (42) and (43). This can be achieved if the NLRT (37) is approximated by

$$
T=\int_{0}^{\infty} \frac{\left\langle x^{2}(t)\right\rangle-\left\langle x^{2}\right\rangle_{s t}}{\left\langle x^{2}(0)\right\rangle-\left\langle x^{2}\right\rangle_{s t}} d t \simeq \int_{0}^{T_{c}} \frac{\left\langle x^{2}(t)\right\rangle-\left\langle x^{2}\right\rangle_{s t}}{\left\langle x^{2}(0)\right\rangle-\left\langle x^{2}\right\rangle_{s t}} d t
$$

where $T_{c}$ is a quench time which guarantees that the system has reached its steady-state value. It can take the value $T_{c}=1.5 T_{0 N L}^{O U}$, with $T_{0 N L}^{O U}$ the same 
as Eq. (43). If we take $\left\langle x^{2}(0)\right\rangle=0$, it can be shown that the $\mathrm{RO}$ can be written as

$$
\mathfrak{R}=\frac{T_{c}-T_{N L}^{O U}}{T_{c}-T_{O N L}^{O U}}=1+\frac{T_{0 N L}^{O U}-T_{N L}^{O U}}{T_{c}-T_{O N L}^{O U}} .
$$

According to Eqs. (42), (43) we now have

$$
\mathfrak{R}=1+\frac{2 e^{-\beta_{M}^{2}}}{2 \mathcal{B} T_{0 N L}^{O U}} \sum_{n=1}^{\infty}[\psi(n+1 / 2)-\psi(1 / 2)] \frac{\left(\beta_{M}^{2}\right)^{n}}{n !},
$$

and

$$
2 \mathcal{B} T_{0 N L}^{O U}=\ln \left(\frac{a x_{s t}^{2}}{2 D_{e}}\right)-\psi(1 / 2)+\frac{3 a \tau}{1-a \tau} .
$$

Therefore, the RO given by Eq. (48) is clearly heat bath memory dependent

\subsection{Numerical simulation}

We now compare the theoretical results with the numerical simulation for the specific OU friction memory kernel. We have employed a unit mass $m=1$ moving in a nonlinear potential with $a_{0}=1, b_{0}=10^{-3}$, so the system relaxes towards the steady state at $x=\left(a_{0} / b_{0}\right)^{1 / 2}$. To implement the simulations, we numerically integrate the pair of stochastic differential equations (31-32) by means of the Heun method [56]. In all cases we have employed a number of independent realizations of the noise $N=10^{6}$, obtaining a relative statistical uncertainty of the order of $2 \times 10^{-4}$ for both MFPT and NLRT. For the temporal discretization we have used a step of typically $\Delta t=3 \times 10^{-3}$, with which the estimated error in the results associated with discretization has been comparable to or lower than the statistical errors

A first series of simulations was performed to check the convergence to the overdamped limit as one increases the friction value. One expects to reach this limit when the time scale $m / \gamma_{0}$ is much smaller that other time scales in the problem, such as $\tau$ and $\gamma_{0} / a_{0}$. For $\tau=1$ and the other used parameters the condition becomes $\gamma_{0} \gg 1$. In Fig. 2 we show the First Passage Time statistics (both mean value and standard deviation) and the corresponding predictions from Eqs. (35), (18), when varying $\gamma_{0}$. We also show the Nonlinear Relaxation Time, compared to the prediction Eq. (42). We see that for $\gamma_{0} \gtrsim 5$ the agreement with theoretical results is very good, with the simulation results converging perfectly to the theoretical predictions.

We next check the results for the MFPT statistics. In Fig. 3 we plot the MFPT as a function of the placement of the absorbing barrier, for $\gamma_{0}=20$ 


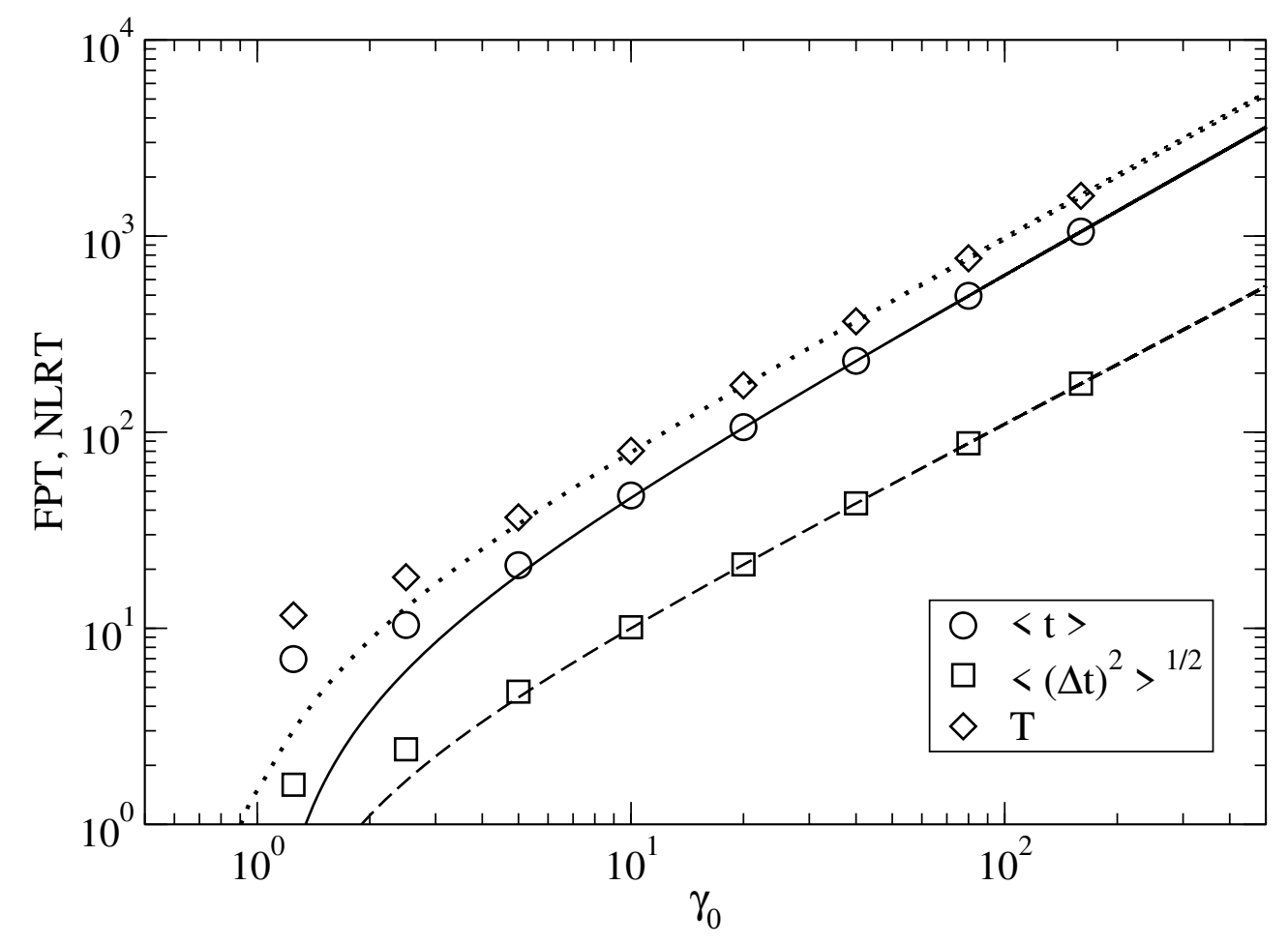

Figure 2: FPT statistics and NLRT vs. $\gamma_{0}$, for $a_{0}=1, b_{0}=10^{-3}, \lambda=10^{-3}, \tau=1, F_{0}=0$. Symbols and lines are simulation results and theoretical predictions, from Eqs. $(35,18,42)$, respectively. Circles and solid line: MFPT $\langle t\rangle$ (with absorbing barriers placed at $x=$ $\pm 1)$; squares and dashed line: standard deviation $\left\langle(\Delta t)^{2}\right\rangle^{1 / 2}$; diamonds and dotted line: Nonlinear Relaxation Time $T$. 


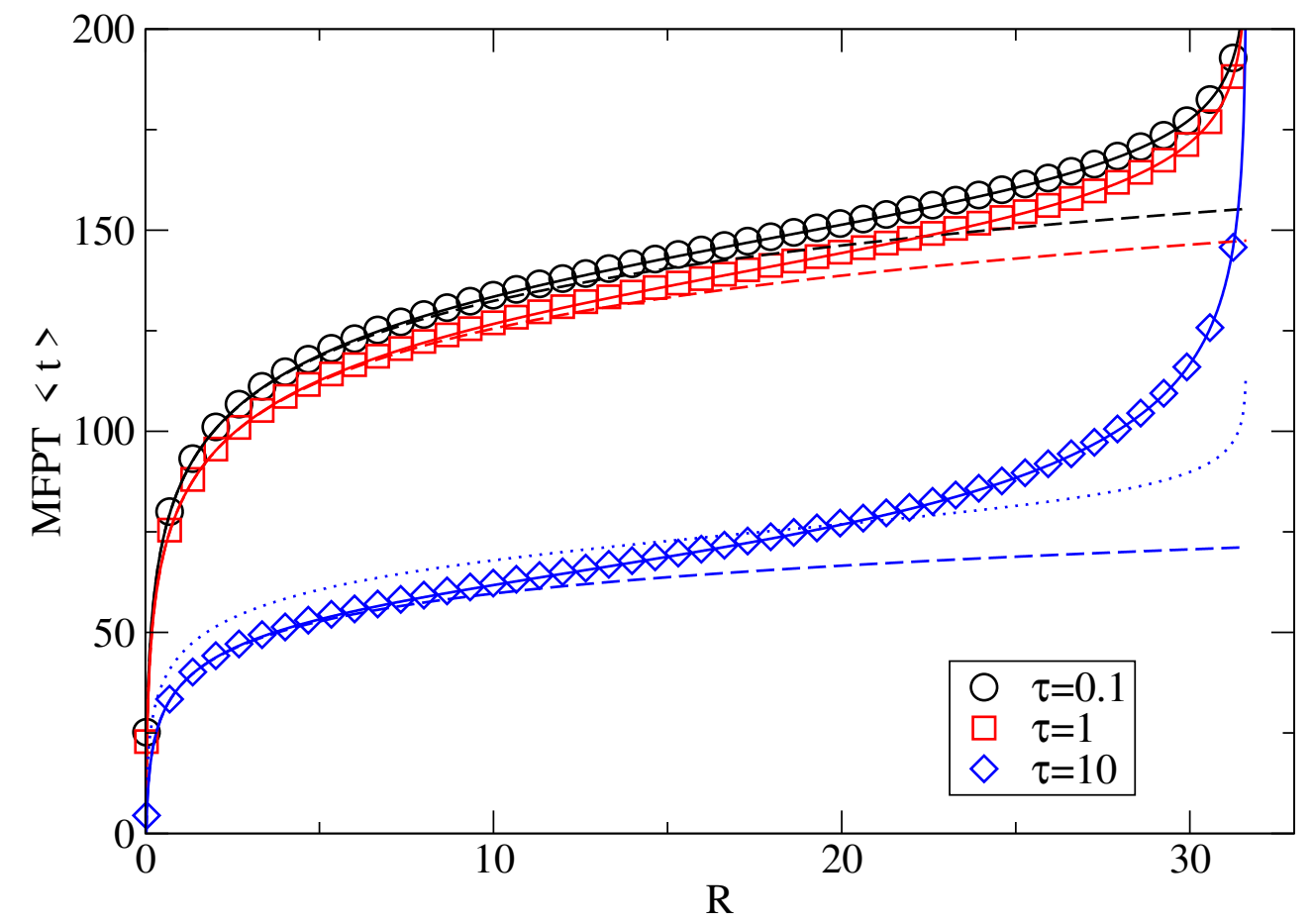

Figure 3: MFPT vs. placement $R$ of the absorbing barrier, for $a_{0}=1, b_{0}=10^{-3}$, $\lambda=10^{-3}, \gamma_{0}=20, F_{0}=0.01$. Symbols and lines indicate simulation and theoretical results respectively. Black lines and circles: $\tau=0.1$, red lines and squares: $\tau=1$, blue lines and diamonds: $\tau=10$. Continuous lines: nonlinear predictions from Eq. (35); dashed lines: linear approximation from Eq. (27); dotted line: nonlinear prediction from Ref. [19] for $\tau=10$. 


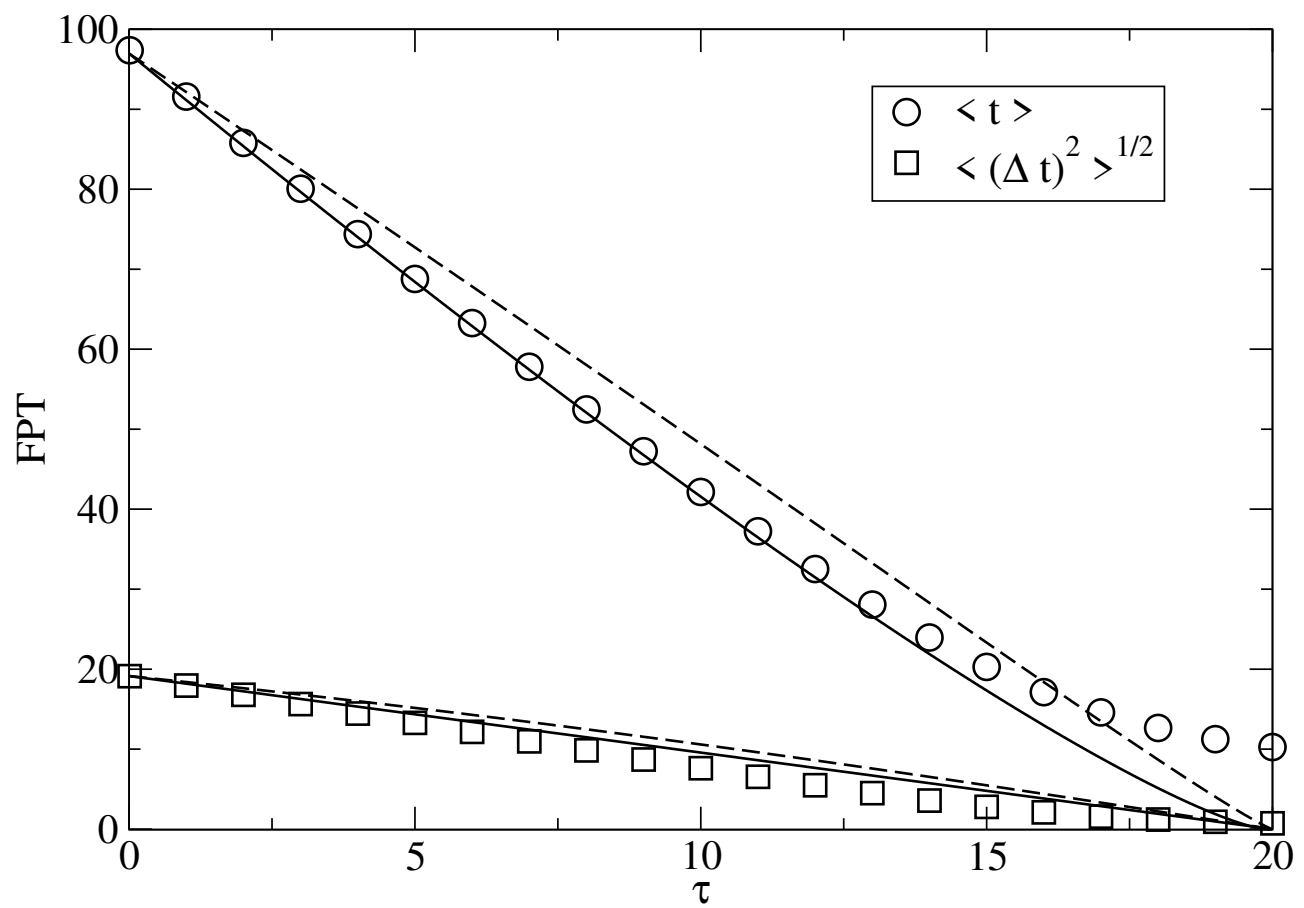

Figure 4: FPT statistics vs. noise time correlation $\tau$, for $a_{0}=1, b_{0}=10^{-3}, \lambda=10^{-3}$, $\gamma_{0}=20, F_{0}=0.01$, and absorbing barriers placed at $x= \pm 1$. Symbols and lines indicate simulation and theoretical results respectively. Circles: MFPT $\langle t\rangle$; squares: standard deviation $\left\langle(\Delta t)^{2}\right\rangle^{1 / 2}$. Continuous lines: nonlinear predictions from Eq. $(35,18)$; dashed lines: predictions from Ref. [19].

and the external field $F_{0}=0.01$. We have employed three values of the correlation time $\tau=0.1,1,10$. For these cases we plot both the linear approximation from Eq. (27) and the nonlinear prediction from Eq. (35). For the case $\tau=10$ we also plot the nonlinear prediction from Ref. [19]. We see that, whereas the linear prediction only works near the unstable state, the nonlinear prediction has a much larger range of validity reaching the neighborhoods of the stable final state. Note however that the prediction diverges at exactly the stable state whereas the simulations provides finite MFPT values at this point. We also see that the nonlinear prediction from Ref. [19] is not very good for not very small correlation times.

The dependence of the predictions on the correlation time of the noise is studied in more detail in the next pair of figures. First, in Fig. 4 the FPT statistics is studied as a function of $\tau$. Here, both MFPT and standard 


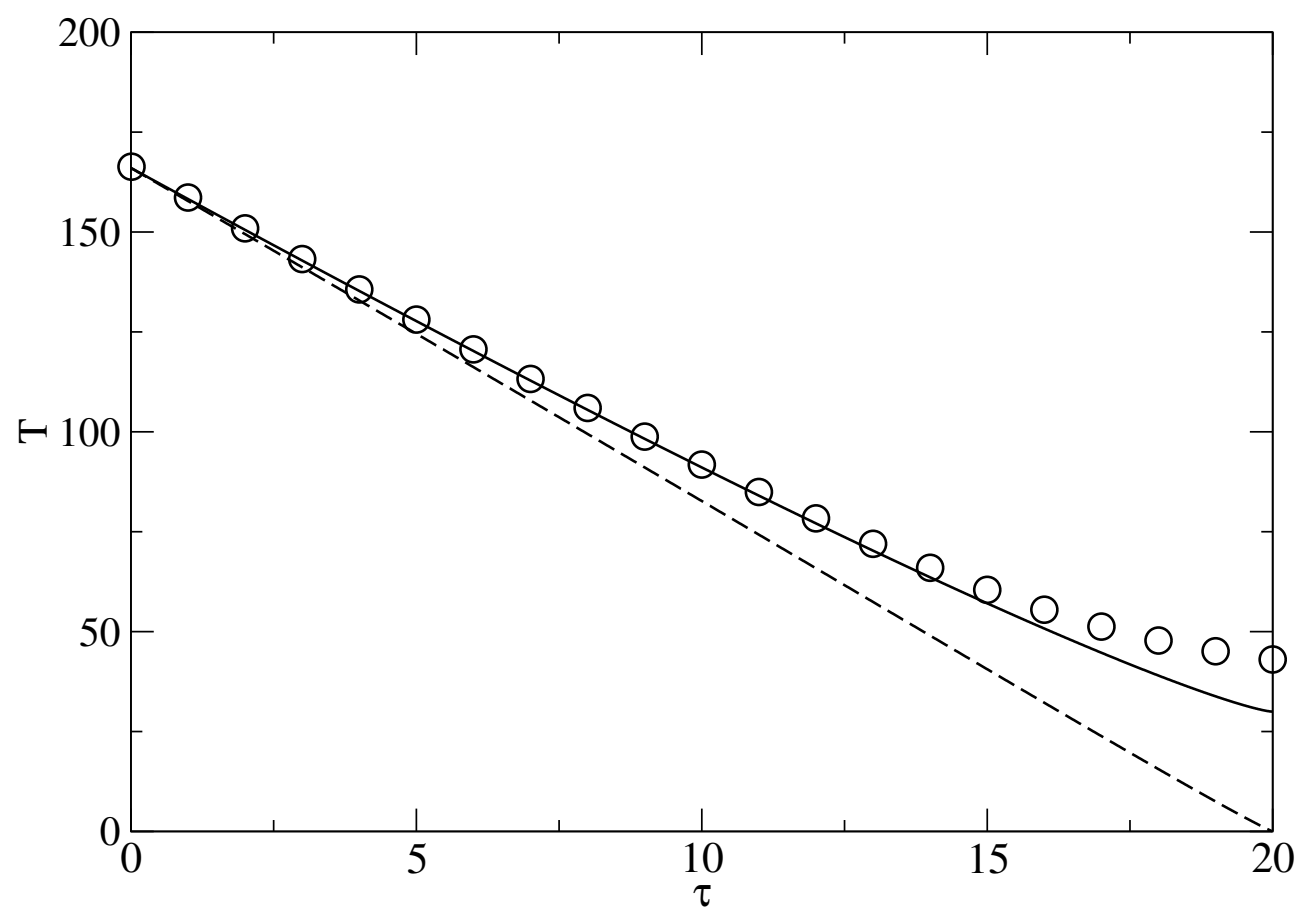

Figure 5: NLRT vs. noise time correlation $\tau$, for $a_{0}=1, b_{0}=10^{-3}, \lambda=10^{-3}, \gamma_{0}=$ 20, $F_{0}=0.01$. Circles: simulation results; continuous lines: nonlinear predictions from Eq. (35); dashed lines: predictions from Ref. [19].

deviation are compared to theoretical predictions from Eq. (35), (18). Theoretical predictions from Ref. [19] are also plotted. In particular the MFPT agrees very well in a large range of values of $\tau$. We also see that results from Ref. [19] predicts the correct Markovian result (at $\tau=0$ ) but the $\tau$ dependence is only qualitatively correct, with increasing differences as $\tau$ is raised. The theoretical results become wrong when approaching $\tau=20$. Note that for our parameters this corresponds to $a \tau=1$, for which our approach breaks down. The dependence on $\tau$ of the standard deviations also shows a fair agreement with theoretical predictions. For this last case we see that results from Eq. (18) are slightly better that those of Ref. [19], although both of them are quite similar.

In Fig. 5 we plot the NLRT versus the correlation time $\tau$. We see that the predictions from Eq. (35) compare very well to the simulation results, showing a correct dependence on $\tau$, except near the $\tau=20$ value. This agreement appears to be better than the prediction of Ref. [19], which only 


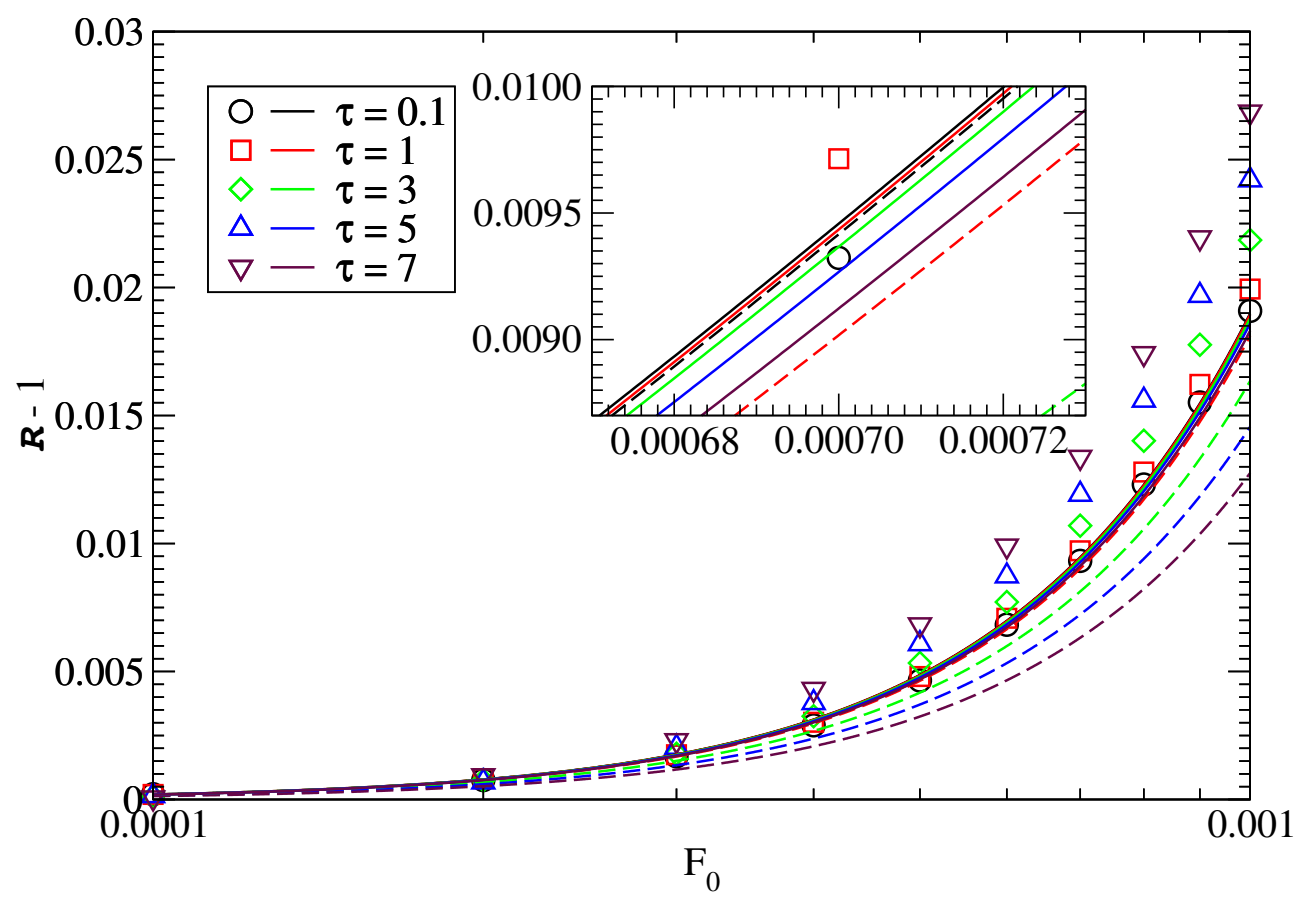

Figure 6: Relative change of RO, $\mathfrak{R}-1$, vs. external force $F_{0}$, for $a_{0}=1, b_{0}=10^{-3}$, $\lambda=10^{-4}, \gamma_{0}=20$, and several values of $\tau$. Symbols: simulation results; continuous lines: theoretical predictions from Eq. (48); dashed lines: predictions from Ref. [19]. In the inset a detailed view around the Markovian limit at $F_{0}=7 \times 10^{-4}$ is shown. Identical colors correspond to the same values of $\tau$.

provides the correct behavior near the Markovian limit.

In Fig. 6 we plot the RO $\mathfrak{R}-1$, versus the external force $F_{0}$, for values of $a_{0}=1, b_{0}=10^{-3}, \lambda=10^{-4}, \gamma_{0}=20$, and several values of $\tau$. We first see that theoretical results of Eq. (48) predict a very weak dependence on $\tau$, so that all curves in a large $\tau$ span almost overlap each other (a more detailed view is shown in the inset). For the smallest external forces we see that simulation results for all $\tau$ also coincide, and only for larger $F_{0}$ the results for the largest $\tau$ values start to separate from theoretical results. In the calculated range of forces, the theoretical result (48) is shown to agree very well for $\tau$ values up to the order of 1.0, but all curves remain very close to the quasi-Markovian result $\tau=0.1$.

We also see that predictions of Ref. [19] start to separate from simulations at smaller values of $F_{0}$. Predictions of Ref. [19] coincide with the numerical simulation close to the Markovian limit, which was an expected result, but 


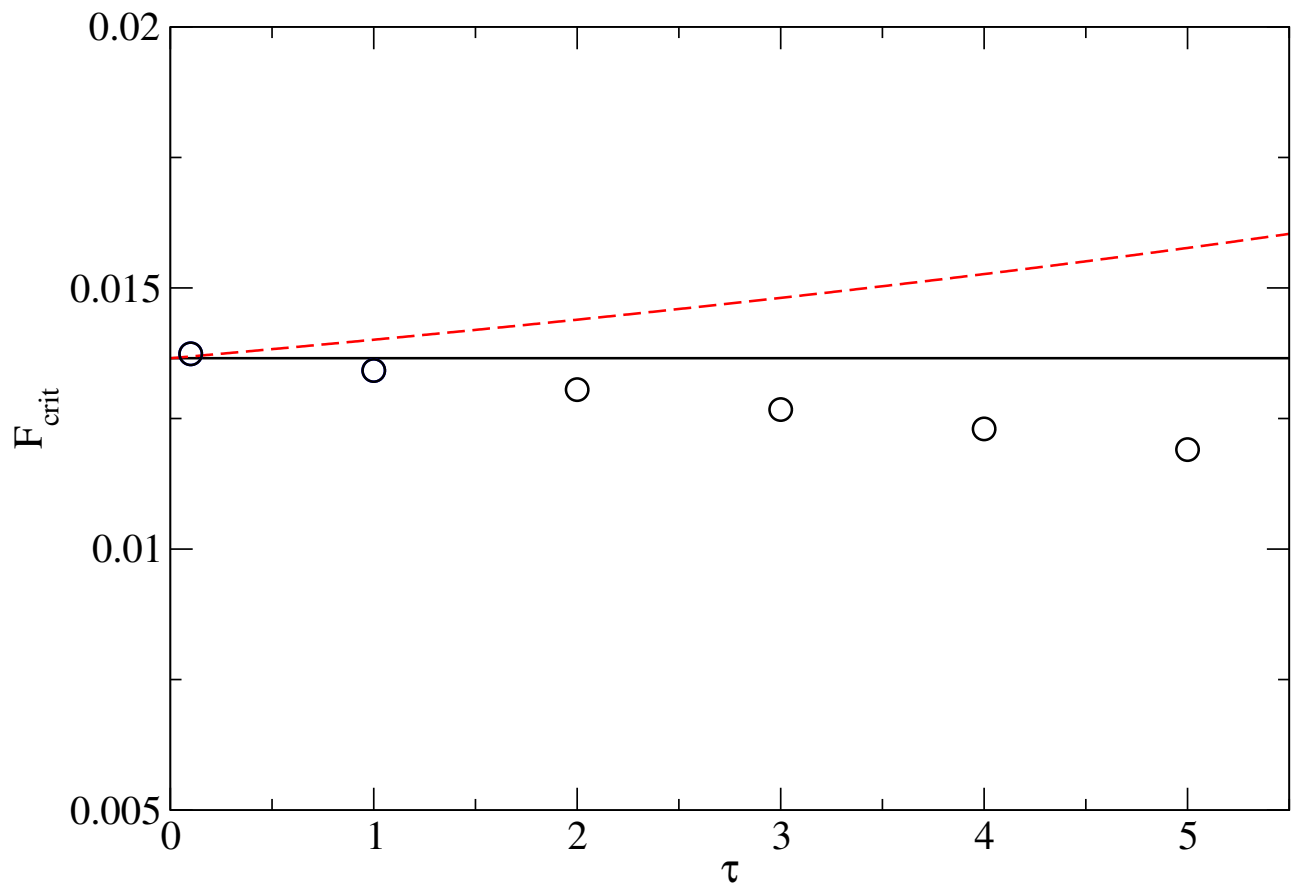

Figure 7: Critical force for detection, according to the criterium related with SFPT in Eq. 45 , vs. $\tau$, for $a_{0}=1, b_{0}=10^{-3}, \lambda=10^{-3}, \gamma_{0}=20$. Simulations have been performed for several values of $F_{0}$ at each $\tau$, in order to find the value at which the change in MFPT was greater than its standard deviation (see text). Circles: simulation results; black continuous line: nonlinear prediction from Eq. (45); red dashed line: prediction from Ref. [19].

when increasing $\tau$ they exhibit a stronger dependence, with the curves moving in the opposite direction than in simulations. In conclusion practically all the curves coincide for forces up to values around $F_{0}=0.0002$, at which a dependence on $\tau$ starts to be built improving $\mathfrak{R}$ in simulation results but with the contrary effect in the predictions of Ref. [19]. On the contrary our current theory remains very close to the Markovian limit. Note that simulation results show that Markovian signals are the most difficult to detect, thus the Markovian case is the optimum one for marking the detector capability.

In Fig. 7 the critical force for detection, according to the SFPT criterion, is represented as a function of the correlation time. For this figure a series of simulations have been performed at each $\tau$ by using several values of $F_{0}$, and finding by interpolation the value $F_{c}$ of external force at which Eq. (44) becomes an equality. We see that simulation results indicate a very weak 
dependence of $F_{c}$, decreasing on $\tau$. Thus, similarly to the Receiver Output results, correlation time improves detection sensibility, and hence the Markovian case marks the operational limits of the detector. Theoretical result of Eq. (45) predicts a $F_{c}$ that does not depend on $\tau$, remaining always equal to the Markovian limit. This improves the prediction of Ref. [19], which predicted a dependence on $\tau$ which results to be opposite to simulations.

\section{Concluding Remarks}

Using the linear overdamped GLE with an arbitrary friction memory kernel, we have been able to characterize, by means of SFPT, the decay process of an unstable state for a charged Brownian particle embedded in non-Markovian heat baths and under the action of an external electric field. The versatility of the QD approach is shown to be effective even for arbitrary friction memory kernel. In this linear regime the particular OU memory kernel is used to calculate the SFPT.

Also we have shown that the OU memory kernel allows to characterize the decay process of the unstable state when the nonlinear contribution of the bistable potential is taken into account. In this case, we exactly calculate the nonlinear SFPT and NLRT. The strategy of solution in this work is new and the theoretical results have been compared with those of numerical simulation showing excellent agreements.

For weak signals detection in OU heat baths, it has been shown that the criterion related to the SFPT is heat bath memory independent, which leads to the same critical value $\beta_{c}=1.36543$ as in the Markovian heat bath. Simulation results indicate a very weak, decreasing dependence on $\tau$, whereas the theoretical results of Ref. [19] the opposite dependence was obtained. A similar situation occurs for the RO, in which theoretical predictions, even being heat bath memory dependent, have an extremely weak dependence on $\tau$. Here, simulation results remain very close to the Markovian limit for small external forces and for not large values of $\tau$ as seen in Fig. 6. Thus for both SFPT and RO criteria the current theory provides results close or equal to the Markovian limit that, without being perfect, still improves the older calculation of previous reference. The Markovian case has been shown to mark the minimum force value at which the signal is guaranteed to be detected regardless of its correlation time.

Finally, our study may be extended to analyze the problem of multiplicative noise [47, 48], the noise enhanced stability (NES) phenomenon 
$[49,50,51,52]$, also the mean switching time to detect weak signals [53], as well as single photon detectors based on Josephson junctions [54].

\section{Acknowledgments}

JIJA thanks to Consejo Nacional de Ciencia y Tecnología (CONACyT, México) for sabbatical grant (Reg:325093/472535). JIJA also deeply thanks to Escuela Superior de Física y Matemáticas of Instituto Politécnico Nacional (ESFM-IPN) for a sabbatical host. NSS thanks support from CONACyT México, COFAA-IPN and EDI-IPN. LR-P thanks support from the Ministerio de Ciencia, Innovacin y Universidades (Spain) and FEDER (European Union), under project PGC2018-095456-B-I00. The authors are grateful to J. M. Sancho for his valuable suggestions and L. Whitehill for his English corrections.

\section{References}

[1] R. Metzler, G. Oshanin, and S. Redner, First-Passage Phenomena and Their Applications, Singapore: World Scientific, 2004.

[2] Redner, S. A guide to First- Passage Processes, Cambridge University Press, Cambridge, England, 2001.

[3] O. Bénichou and R. Voituriez, From first-passage times of random walks in confinement to geometry, controlled kinetics Phys. Rep. 539 (2014) 225

[4] C. Hohenegger, R. Durr, and D.M. Senter, Mean first passage time in a thermally fluctuating viscoelastic fluid, J. Non-Newton Fluid Mech. 242 (2017) 48.

[5] D. S. Grebenkov, First Exit Times of Harmonically Trapped Particles: A Didactic Review, J. Phys. A: Math. Theor. 48 (2015) 013001.

[6] S. B. Yuste, G. Oshanin, K. Lindenberg, O. Bénichou, and J. Klafter, Survival probability of a particle in a sea of mobile traps: A tale of tails, Phys. Rev. E 78 (2008) 021105.

[7] L. P. Sanders and T. Ambjornsson, First passage times for a tracer particle in single file diffusion and fractional Brownian motion, J. Chem. Phys. 136 (2012) 175103 . 
[8] J. H. Jeon, A. V. Chechkin, and R. Metzler, First passage behaviour of fractional Brownian motion in two-dimensional wedge domains, Europhys. Lett. 94 (2011) 20008.

[9] V. Tejedor, o. Bénichou, R. Metzler, and R. Voituriez, Residual mean first-passage time for jump processes: theory and applications to Lévy flights and fractional Brownian motion, J. Phys. A 44 (2011) 255003.

[10] T. Koren, J. Klafter, and M. Magdziarz, First passage times of Lévy flights coexisting with subdiffusion, Phys. Rev. E 76 (2007) 031129.

[11] D.Holcman, A.Marchewka, and Z. Schuss, Survival probability of diffusion with trapping in cellular neurobiology, Phys. Rev. E 72 (2005) 031910 .

[12] O. Bénichou, C. Chevalier, J. Klafter, B. Meyer, and R. Volturiez, Geometry-controlled kinetics, Nat. Chem. 2 (2010) 472.

[13] Chuang Yi, On the first passage time distribution of an OrnsteinUhlenbeck process, Quantitative Finance, 10 (2010), 957

[14] L. Alili, P. Patie, and J. L. Pedersen, Representations of the First Hitting Time Density of an Ornstein-Uhlenbeck Process, Stoch. Models 21 (2005) 967.

[15] A. E. Cardenas and R. Elber, Markovian and Non-Markovian Modeling of Membrane Dynamics with Milestoning, J. Phys. Chem. B 120 (2016) 8208 .

[16] S. Kou and X. S. Xie, Generalized Langevin Equation with Fractional Gaussian Noise: Subdiffusion within a Single Protein Molecule Phys. Rev. Lett. 93, (2004) 180603.

[17] T. Guérin, N. Levernier, O. Bénichou, and R. Voituriez, Mean firstpassage times of non-Markovian random walkers in confinement, Nature 534 ( 2016) 356.

[18] O. Bénichou, T. Guérin, and R. Volturiez, Mean first-passage times in confined media: from Markovian to non-Markovian processes, J. Phys. A: Math. Theor. 48 (2015) 163001. 
[19] J. I. Jiménez-Aquino, R. M. Velasco, and M. Romero-Bastida, Detection of weak signals in memory thermal baths, Phys. Rev. E 90 (2014) 052146

[20] M. Nyberg, Tobias Ambjornsson, and Ludving Lizana, A simple method to calculate first-passage time densities with arbitrary initial conditions, New J. Phys. 18 (2016) 063019.

[21] T. Srokowski, Bistable generalised Langevin dynamics driven by correlated noise possessing a long jump distribution: barrier crossing and stochastic resonance, Eur. Phys. J. B. 86 (2013) 239.

[22] J. M. Sancho and M. San Miguel, Passage times for the decay of an unstable state triggered by colored noise, Phys. Rev. A 39 (1989) 2722.

[23] L. Ramirez-Piscina, J. M. Sancho, F. J. De la Rubia, K. Lindenberg, and G. P. Tsironis, First-passage time in bistable potential with colored noise, Phys. Rev. A 40 (1989) 2120.

[24] J. M. Sancho, F. Sagues, and M. San Miguel, Mean first-passage time of continuous non-Markovian processes driven by colored noise, Phys. Rev. A 33 (1986) 3399 .

[25] J. Masoliver, K. Lindenberg, and B. J. West, First-passage times for non-Markovian processes: Correlated impacts on bound processes, Phys. Rev. A 34 (1986) 2351.

[26] P. Hanggi and P. Talkner, First-passage time problems for nonMarkovian processes, Phys. Rev A 32 (1985) 1934.

[27] A. O. Bolivar, Non-Markovian effects on the Brownian motion of a free particle, Physica A 390 (2011) 3095?3107.

[28] T. Verechtchaguina, I. M. Sokolov and L. Schimansky-Geier, First passage time densities in non-Markovian models with subthreshold oscillations, Europhys. Lett., 73 (2006) 691.

[29] A. Fiasconaro and B. Spagnolo, Stability measures in metastable states with Gaussian colored noise, Physical Review E 80 (2009) 041110.

[30] R. Kubo, The fluctuation-dissipation theorem, Rep. Progr. Phys. 29 (1966) 255. 
[31] G. Vemuri and R. Roy, Super-regenerative laser receiver: Transient dynamics of a laser with an external signal, Phys. Rev. A 39 (1989) 2539.

[32] J. I. Jiménez-Aquino and J. M. Sancho, Nonlinear relaxation time and the detection of weak signals, Phys. Rev. A 43 (1991) 589.

[33] S. Balle, F. De Pasquale, and M. San Miguel, Passage-time calculation for the detection of weak signals via the transient dynamics of a laser, Phys. Rev. A 41 (1990) 5012.

[34] I. Littler, S. Balle, K. Bergmann, G. Vemuri, and R. Roy, Detection of weak signals via the decay of an unstable state: Initiation of an injection-seeded laser, Phys. Rev. A 41 (1990) 4131.

[35] J. I. Jiménez-Aquino and M. Romero-Bastida, Detection of weak signals through nonlinear relaxation times for a Brownian particle in an electromagnetic field, Phys. Rev. E 84 (2011) 011137.

[36] F. de Pasquale and P. Tombesi, The decay of an unstable equilibrium state near a "critical point", Phys. Lett. A 72 (1979) 7.

[37] F. Haake, J. W. Haus, and R. Glauber, Passage-time statistics for the decay of unstable equilibrium states, Phys. Rev. A 23 (1981) 3255.

[38] W. Nadler and K. Schulten, Generalized moment expansion for Brownian relaxation processes, J. Chem. Phys. 82 (1985) 151.

[39] J. Casademunt, J. I. Jiménez-Aquino, and J. M. Sancho, Decay of unstable states in the presence of colored noise and random initial conditions. I. Theory of nonlinear relaxation times, Phys. Rev. A 40 (1989) 5905.

[40] N. V. Agudov and A. N. Malakhov, Decay of unstable equilibrium and nonequilibrium states with inverse probability current taken into account, Phys. Rev. E 60 (1999) 6333.

[41] N. V. Agudov and B. Spagnolo, Noise-enhanced stability of periodically driven metastable states, Phys. Rev. E 64 (2001) 035102

[42] A. N. Malakhov and A. L. Pankratov, Influence of thermal fluctuations on time characteristics of a single Josephson element with high damping exact solution, Physica C 269 (1996) 46 
[43] A. L. Pankratov, Time evolution of averages in dynamical systems driven by noise, Phys. Lett. A 255 (1999) 17

[44] A. N. Malakhov and A. L. Pankratov, Evolution Times of Probability Distributions and Averages. Exact Solutions of the Kramers' Problem, Adv. Chem. Phys. 121 (2002) 357.

[45] A. L. Pankratov and B. Spagnolo, Suppression of Timing Errors in Short Overdamped Josephson Junctions, Phys. Rev. Lett. 93 (2004) 177001.

[46] F. de Pasquale, P. Tartaglia and P. Tombesi, Stochastic dynamic approach to the decay of an unstable state, Z. Physik B 43 (1981) 353.

[47] F. de Pasquale, J. M. Sancho, M. San Miguel, and P. Tartaglia Decay of an unstable state in the presence of multiplicative noise, Phys. Rev. A 33 (1986) 4360.

[48] S. Ciuchi, F. de Pasquale, and B. Spagnolo, Nonlinear relaxation in the presence of an absorbing barrier, Phys. Rev. E 47 (1993) 3915.

[49] N. V. Agudov, A. A. Dubkov, and B. Spagnolo, Escape from a metastable state with fuctuating barrier, Physica A 325 (2003) 144.

[50] A. Fiasconaro, B. Spagnolo, and S. Boccaletti, Signatures of noiseenhanced stability in metastable states, Phys. Rev. E 72 (2005) 061110.

[51] Anna V. Gordeeva, A. L. Pankratov, and B. Spagnolo, Noise inducced phenomena in point Josephson junctions, Int. J. Bifurcat. Chaos Vol. 18(9) (2008) 2825.

[52] G. Augello, D. Valentia, and B. Spagnolo,. Non-Gaussian noise effects in the dynamics of a short overdamped Josephson junction, Eur. Phys. J. B 78 (2010) 225.

[53] Anna V. Gordeeva and A. L. Pankratov, Minimization of timing errors in reproduction of single flux quantum pulses, Appl. Phys. Lett. 88 (2006) 022505

[54] G. Oelsner, C. K. Andersen, M. Rehák, M. Schmelz, S. Anders, M. Grajcar, U. Hubner, K. Molmer, and E. Il'ichev, Detection of Weak Microwave Fields with an Underdamped Josephson Junction, Phys. Rev. Appl. 7 (2017) 014012 
[55] M. Abramowitz and I. A. Stegun, Handbook of Mathematical Functions, Dover, New York, 1972.

[56] T.C. Gard, Introduction to Stochastic Differential Equations, of Monographs and Textbooks in Pure and Applied Mathematics, Vol 114, Marcel Dekker, New York, 1988. 